\title{
Les sources de lumière dans le bleu et le proche UV basées sur les diodes à semiconducteurs : état actuel de l'art et perspectives
}

\author{
J.L. Deiss
}

Institut de Physique et Chimie des Matériaux de Strasbourg, Groupe d'Optique

Nonlinéaire et d'Optoélectronique, Unité Mixte 380046, CNRS-ULP-EHICS, 23 rue du

Loess, BP. 20 CR, 67037 Strasbourg cedex, France

\begin{abstract}
Résumé : Les diodes à semiconducteurs émettrices de lumière connaissent un regain d'activité grâce à leurs applications potentielles dans divers domaines en pleine expansion, allant de l'affichage en couleurs au stockage optique de données. Ces développements reposent sur la mise au point de diodes émettant dans le bleu et basées sur 1'utilisation de composés à plus large bande interdite, tels ZnSe ou GaN. Dans cet article, on rappelle le principe de fonctionnement de ces diodes et les progrès réalisés à la fois dans leur conception et dans leur fabrication.
\end{abstract}

\section{INTRODUCTION}

Les diodes à semiconducteurs émettrices de lumière dans la gamme spectrale allant de l'infrarouge au vert sont déjà largement utilisées et commercialisées depuis de nombreuses années (environ 1960). Ce type de composants optoélectroniques prend une importance croissante dans des applications très diverses allant de l'affichage en couleurs sur écrans plats à la transmission et au stockage optique des données [1-3]. Parmi ces composants, on distingue entre les diodes électroluminescentes (DEL) ou les diodes lasers (DL), qui sont respectivement des sources de lumière incohérente ou cohérente.

Actuellement, les diodes sont principalement réalisées à partir des semiconducteurs binaires III-V à faible bande interdite (ex. GaAs) et de leurs alliages respectifs: elles émettent généralement dans la partie infrarouge (IR) et rouge (R) du spectre électromagnétique. Les dispositifs basés sur ces composants trouvent leurs principales applications dans les télécommunications par fibres optiques et la lecture de disques compacts.

L'utilisation de composés ternaires (ex. AlGaAs) ou quaternaires (ex. InGaAlP) a permis d'étendre l'émission de lumière de ces composants jusque dans le vert, en jouant sur la composition des alliages. La technologie de fabrication de ces matériaux III-V en multicouches minces et ultraminces est actuellement bien maîtrisée et permet de réaliser des composants optoélectroniques de très bonne qualité. On dispose à présent de diodes émettrices DEL ou DL superbrillantes, à rendement et durée de vie élevés et émettant de l'infrarouge au vert (de 1500 à $550 \mathrm{~nm}$ ).

Le longueur d'onde $(\lambda)$ de la lumière émise (ou couleur) par ces diodes dépend de la largeur $\mathrm{E}_{\mathrm{g}}$ de la bande interdite du matériau ou du type de dopants utilisé. Par conséquent, l'extension de l'émission de ces diodes vers le bleu $(\lambda<500 \mathrm{~nm})$ ou le proche UV $(\lambda<400$ nm) nécessite l'utilisation de semiconducteurs à bande interdite plus large. Les composés semiconducteurs II-VI ( $\mathrm{ZnSe}, \mathrm{ZnS}$ ) ou les nitrures III-V (GaN, AIN) répondent à ce critère et 
apparaissent comme les matériaux de choix pour la réalisation de ces futures diodes émettant vers les plus courtes longueurs d'onde.

Mais, le développement de composants optoélectroniques basés sur ces composés à large bande interdite a été fortement retardé du fait de sérieux problèmes rencontrés dans l'élaboration de couches minces de ces matériaux. Cependant depuis 1988, des progrès substantiels ont néanmoins été réalisés dans la croissance de ces couches minces de ZnSe ou GaN en utilisant les techniques d'épitaxie hors équilibre (MBE et MOVPE), qui ont contribué au succès des composants à base de GaAs. En 1991, ces progrès ont enfin permis à un groupe de $3 \mathrm{M}$ Corporate Research Laboratories (USA) de démontrer pour la première fois le fonctionnement d'une diode laser bleue à base de $\mathrm{ZnSe}$ [4].

On se propose dans cet article, de faire le point sur l'état actuel du sujet, en décrivant le principe de fonctionnement de ces diodes et en donnant leurs caractéristiques essentielles [5-6]. On indiquera également leurs perspectives de développement, liées à la mise au point de nouvelles structures quantiques et à l'utilisation de matériaux à plus large bande interdite [7-8].

\section{LES DIODES ELECTROLUMINESCENTES}

Le fonctionnement des diodes émettrices de lumière DEL ou DL est basé sur une excitation électronique avec création de porteurs libres par injection électrique, suivie d'une recombinaison radiative de ces porteurs [5]. La structure de base de ces diodes à semiconducteurs est une jonction pn polarisée dans le sens direct (Fig. 1), où:

- les électrons (ou trous) qui sont les porteurs majoritaires dans la région $n$ (ou $p$ ) sont alors injectés dans la région $\mathrm{p}$ (ou $\mathrm{n}$ ), où ils sont minoritaires

- ces électrons (ou trous) excédentaires dans ces régions neutres $p$ (ou $n$ ) diffusent et se recombinent de façon radiative ou non radiative

- si la recombinaison est radiative, la jonction émet un rayonnement: cette émission peut être de nature spontanée (cas des DEL) ou stimulée (pour les DL)

Les semiconducteurs sont choisis sur le critère déterminant d'une recombinaison du type essentiellement radiative des porteurs (électrons ou trous). Par conséquent, la fabrication de DEL performantes exige:

- le choix de semiconducteurs à bande interdite directe (ex. GaAs), où la recombinaison radiative des paires électrons-trous est environ mille fois plus probable que dans les semiconducteurs à gap indirect (ex. Si ou Ge)

- un niveau de dopage élevé dans les régions $n$ et $p$, afin d'augmenter le nombre de porteurs injectés, et donc l'intensité lumineuse émise

- l'élaboration de matériaux en couches minces de haute qualité cristalline, car la présence d'un trop grand nombre de défauts favorise les recombinaisons non radiatives

\subsection{Diodes électroluminescentes à simple homojonction}

Le principe de fonctionnement d'une telle diode est représenté Fig. 1. La jonction pn est une simple homojonction, c.à.d une jonction formée de deux semiconducteurs de même nature mais de dopages différents. La polarisation directe de la diode réduit notablement la barrière de potentiel formée à la jonction, favorisant ainsi l'injection des électrons (ou trous) majoritaires dans les régions voisines $\mathrm{p}$ (ou $\mathrm{n}$ ) où ils se recombinent, en émettant de la lumière (Fig.5). La région $\mathrm{p}$ de la diode (la région active) est la plus radiative car, du fait de la mobilité plus elevée 
des électrons le taux d'injection des électrons dans la région p est plus grand que le taux d'injection des trous dans la région $\mathbf{n}$.

Les DEL sont des sources de lumière incohérente, où domine l'émission spontanée: la longueur d'onde du rayonnement émis dépend de la largeur de la bande interdite du semiconducteur. Les diodes DEL sont caractérisées par leur longueur d'onde d'émission, par leur rendement quantique externe, par leur brillance et aussi par leur durée de vie en fonctionnement. Une DEL peut fonctionner en régime continu ou pulsé (fréquence de coupure $\sim 100 \mathrm{Mhz}$ ).

Les premières diodes à homojonction réalisées à partir de 1965 présentaient un rendement faible et une brillance peu élévée, de plus l'émission de ces diodes était limitée à la région rouge du spectre visible. Exemples:

- les diodes GaAs dopées au Si ou au Zn, émettent dans l'IR à 1000 ou $900 \mathrm{~nm}$

- l'émission bleue est obtenue actuellement par des diodes au SiC dopé $\mathrm{n}$ et $\mathrm{p}$, mais leur rendement est mauvais $(0.1 \%)$ et leur brillance est très faible ( 0.04 lumens/watt)

Ces DEL de faible brillance et limitées principalement à la couleur rouge n'ont servi au départ que comme indicateurs lumineux ou alphanumériques dans les appareils électroniques ou les calculatrices de poche.
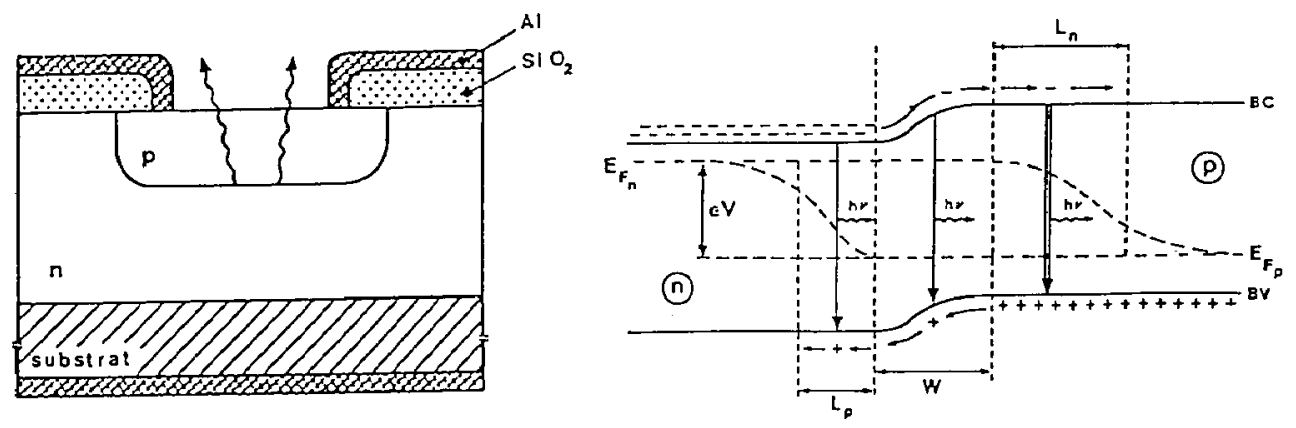

Fig. 1: Structure et fonctionnement d'une diode électroluminescente

\subsection{Diodes électroluminescentes à double hétérostructure}

Les DEL actuelles sont principalement réalisées à partir des composés binaires III-V (type $\mathrm{GaAs})$ et de leurs alliages repectifs $\left(\mathrm{GaAl}_{\mathrm{x}} \mathrm{As}_{1-\mathrm{x}}, \mathrm{GaInAsP} \ldots\right)$ : leurs performances (rendement,brillance...) ont été sensiblement améliorées, grâce:

- à la réduction de l'épaisseur $\mathrm{d}(<1 \mu \mathrm{m})$ de la région active $\mathrm{p}$, assurant ainsi un meilleur confinement des porteurs

- à l'utilisation de structures multicouches de différents semiconducteurs (simple ou double hétérostructure)

- aux progrès sensibles réalisées en science des matériaux qui ont permis la croissance de couches minces épitaxiées III-V de haute qualité cristalline (densité de défauts $<10^{4} \mathrm{~cm}^{-3}$ ) La structure actuelle d'une DEL est plus complexe que celle de la diode à homojonction précédente, elle résulte de la superposition de semiconducteurs différents formant une hétérostructure simple ou double (Fig.2). Ces nouvelles structures permettent d'une part, d'adapter les différentes couches entre elles pour réduire les effets nuisibles d'interfaces et 
d'autre part, d'assurer un meilleur confinement des porteurs et des photons. Le confinement optique des photons est assuré grâce à un abaissement de l'indice de réfraction autour de la zone active.

Une diode typique à double hétérostructure $\mathrm{GaAsP} / \mathrm{GaP}$ est schématisée (Fig.2). Elle est constituée d'un substrat $\mathrm{GaP}(\mathrm{n})$ qui sert de support à une double couche tampon en $\mathrm{GaAsP}$ et à la zone active $\left(\mathrm{GaAs}_{0.35} \mathrm{P}_{0.65}\right)$. Le rôle de ces deux couches tampons est de réaliser une bonne interface entre le substrat et la couche active, permettant ainsi de diminuer la densité des défauts dans la jonction.

L'utilisation d'alliages ternaires de nature différente (ex. GaAlAs, GaAsP, GaInP, AlInP) ou de composition (x) différente (ex. $\mathrm{GaAs}_{\mathrm{x}} \mathrm{P}_{1-\mathrm{x}}$ ) a permis d'étendre l'émission de lumière de ces diodes de l'infrarouge (IR) au vert (V), voir Fig.3.

Les DEL actuelles présentent des brillances élevées jusqu'à 20 lumens/watt ou plus et des rendements atteignant $30 \%$ avec des durées de vie supérieures à 10.000 heures [1].

En 1991, une DEL superbrillante basée sur InGaAlP, émettant dans le rouge ou le vert a été commercialisée par Purdy Electronics, elle est caractérisée par une brillance cinq fois supérieure aux diodes GaAlAs. Ces DEL superbrillantes, du fait de leur visibilité élevée du rouge au vert, permettent des applications nouvelles allant de l'affichage couleur, aux feux de signalisation pour les voitures et le trafic routier.
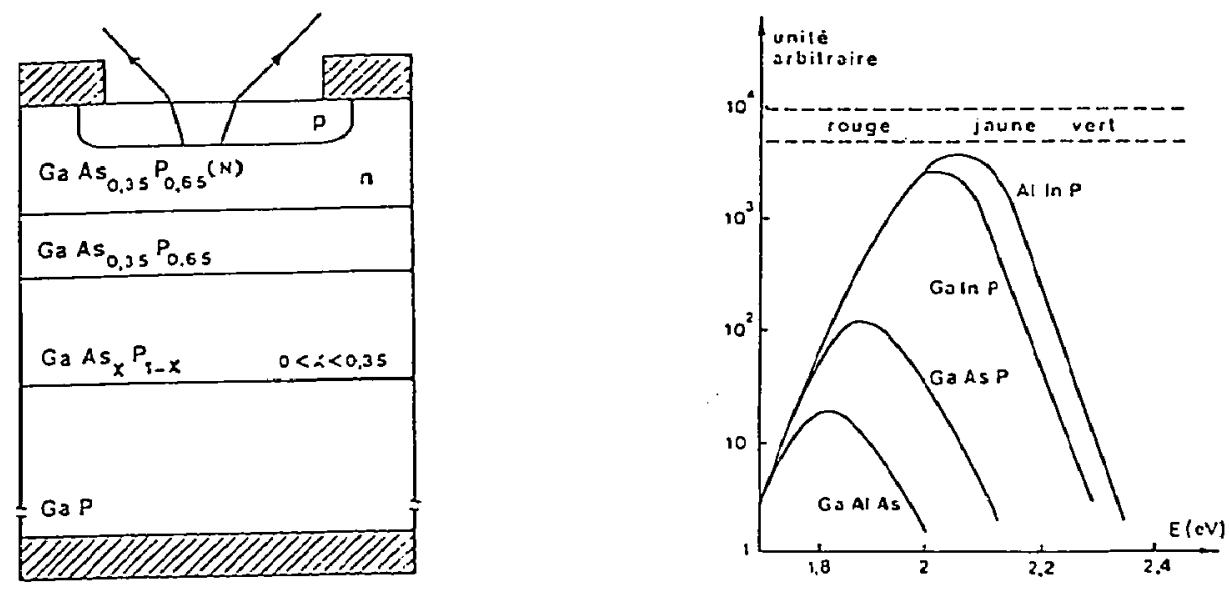

Fig. 2: Diode électroluminescente à double hétérostructure Fig. 3: Spectres d'émission de différentes diodes

\subsection{Diodes électroluminescentes dans le bleu ou UV}

Il existait déjà des diodes fabriquées à partir de $\mathrm{SiC}$, émettant dans le bleu à $490 \mathrm{~nm}$, mais elles présentaient une brillance très faible. Le développement de diodes plus performantes, émettant à plus courtes longueurs d'onde, passe donc impérativement par l'utilisation de semiconducteurs à plus large bande interdite, tels les composés II-VI ( $\mathrm{ZnSe}, \mathrm{ZnS}$...) ou les nitrures III-V (GaN, AlN...).

Les progrès récents (1990) dans l'élaboration de ces couches minces ont permis d'obtenir des DEL superbrillantes dans le bleu [8]. 
- en 1993, la Société Nichia Co. (Japon) a commercialisé une diode basée sur GaN émettant à $450 \mathrm{~nm}(10 \mathrm{~mA}, 1 \mathrm{~mW})$ avec un rendement de $4 \%$ : cette diode est 100 fois plus intense que les diodes $\mathrm{SiC}$ actuelles [9].

- en 1994, les Sociétés Cree et Eagle Pichler Ind. ont aussi développé des diodes superbrillantes dans le Vert et Bleu basées respectivement sur InGaN et $\mathrm{ZnSe}$ [1]. Exemples:

- la diode $\mathrm{ZnSe} / \mathrm{ZnTeSe}$ émet à $512 \mathrm{~nm}(10 \mathrm{~mA}, 1.3 \mathrm{~mW}, 5,3 \%)$ avec 18 lumens/watt

- la diode $\mathrm{ZnSE} / \mathrm{ZnCdSe}$ émet à $489 \mathrm{~nm}(10 \mathrm{~mA}, 0.3 \mathrm{~mW}, 1.3 \%)$ et 1.7 lumens/watt

\section{LES LASERS A SEMICONDUCTEURS OU DIODES LASERS}

Les diodes lasers (DL) sont aussi réalisées à partir d'une jonction pn. Ce sont des sources de lumière cohérente basées sur l'émission stimulée. Cette émission stimulée de lumière est à l'origine de l'effet laser et donne un faisceau lumineux directif, cohérent et très brillant. Pour faire fonctionner une diode électroluminescente (DEL) en diode laser, il suffit d'augmenter le courant (c.à.d l'injection de porteurs) pour réaliser la nécessaire condition d'inversion de population (Fig.4).

\subsection{Effet laser}

La spécificité de la structure des bandes d'énergie des semiconducteurs (Fig.5), par rapport aux niveaux atomiques discrets des lasers à gaz, impose ici deux conditions pour obtenir un effet laser dans ces diodes, à savoir:

- une condition d'inversion de population $\left(\mathrm{N}_{2}>\mathrm{N}_{1}\right)$, caractérisée par un coefficient d'absorption $\alpha<0$, c.à.d un gain $g(E)=-\alpha>0$

- une condition d'amplification qui exige que le gain $g(E)$ soit supérieur aux pertes dans la zone active (la cavité optique), ceci est réalisée si le courant $J>J_{s}$ (courant seuil)

Les pertes sont dues à des absorptions et diffusions des photons dans le milieu amplificateur et également aux réflectivités partielles dans la cavité.

La condition d'inversion est réalisée si le coefficient d'absorption $\alpha$ et la probabilité d'émission stimulée $P_{\text {st }}$ sont négatifs, or:

$$
\alpha=B_{12}\left(N_{1}-N_{2}\right) h v / c \Delta V \quad \text { et } \quad P_{s t}=P_{s p}[1-\exp (E-\Delta F / k T)]
$$

D'après les relations (1), la condition d'inversion exige donc, d'une part que $\mathrm{N}_{2}>\mathrm{N}_{\mathrm{l}}$ (il y amplification de lumière dans le milieu) et d'autre part que $\Delta \mathrm{F}=\mathrm{E}_{\mathrm{Fc}}-\mathrm{E}_{\mathrm{Fv}}>0$

Or, la condition $\Delta F>0$ impose que $\Delta F>E g$, c.àd que les pseudo niveaux de Fermi $E_{F c}$ et $E_{F v}$ doivent être situés ici dans les bandes de valence et de conduction

On satisfait à ces conditions d'effet laser dans les jonctions pn, grâce à:

- un niveau de dopage $n$ et $p$ très élevé (semiconducteurs dégénérés), ce qui entraîne le déplacement des pseudo-niveaux de Fermi $\mathrm{E}_{\mathrm{Fe}}$ et $\mathrm{E}_{\mathrm{Fv}}$ à l'intérieur des bandes de conduction et de valence (Fig.5)

- une augmentation du courant injecté $\mathbf{J}$ au-delà du courant de transparence $\mathbf{J}_{0}$ (défini par $\alpha=0$ ): à partir de $J_{0}$ l'émission stimulée croît brutalement (Fig.4)

- ensuite une augmentation supplémentaire du courant $\mathrm{J}>\mathrm{J}_{\mathrm{s}}$ (courant seuil) permet de compenser les pertes dans la cavité et le laser oscille 


\subsection{Diodes lasers classiques}

Ces diodes lasers (DL) sont construites à partir d'une simple homojonction ou à partir d'une hétérojonction simple ou double, polarisée en direct.

On optimise les performances (rendement, brillance...) de ces diodes DL en agissant sur les paramètres caractéristiques du laser à semiconducteurs, à savoir: le courant seuil $\mathrm{J}_{\mathrm{s}}$, le gain différentiel $A_{j}$ et le facteur $\Gamma$ de confinement optique des photons [6]. Le gain différentiel correspond à la pente de l'émission stimulée et le facteur $\Gamma$ est une mesure du confinement des photons dans la cavité optique (c'est la fraction des photons confinés).

\subsubsection{Diodes lasers à homojonction}

Ces diodes lasers, obtenues à partir d'une simple homojonction, sont plus faciles à fabriquer par simple croissance et diffusion d'une couche $p$ sur un substrat dopé n (Fig.6). L'émission laser est confinée dans une région étroite de la jonction (la zone active). La divergence du faisceau est importante par suite de la diffraction par cette fente étroite. Ces diodes lasers sont caractérisées par un rendement faible et un courant seuil élevé $\left(\mathrm{J}_{\mathrm{s}} \sim 30.000 \mathrm{~A} / \mathrm{cm}^{2}\right)$.

Le confinement partiel des photons dans la zone active est dû ici, à une variation d'indice résultant du dopage élevé et du confinement des électrons et trous dans cette région: ceci entraîne une réflexion interne des photons émis (on a un guidage de l'onde). Mais comme ces différences d'indice ne sont pas importantes, une partie des photons de la cavité est transmise à l'extérieur: le rendement du laser en est donc affecté.

Les dimensions typiques de ces diodes à homojonction sont: $\mathrm{L}=100 \mu \mathrm{m}, 1=10 \mu \mathrm{m}$ et l'épaisseur de la zone active $\mathrm{d}=\sim 0.5 \mu \mathrm{m}$ [Fig.6].

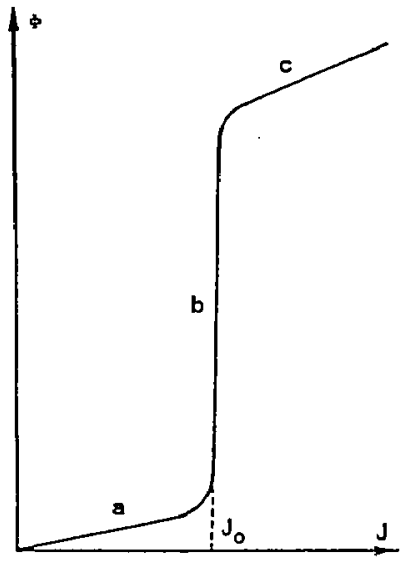

Fig. 4: Intensité émise en fonction du courant a) émission spontanée b) stimulée c) oscillation laser

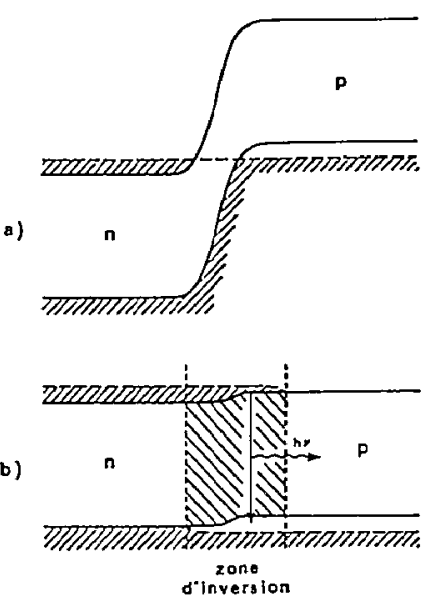

Fig. 5: Bandes d'énergie au voisinage de la jonction a) non polarisée b) polarisée en direct 


\subsubsection{Diodes lasers à double hétérojonction}

La structure réelle des diodes lasers actuelles est en fait plus complexe que celle d'une simple diode à homojonction. L'objectif poursuivi est de minimiser le courant seuil $\mathrm{J}_{8}$, par:

- un confinement amélioré des électrons et des trous dans une plus faible section

- un meilleur confinement des photons dans un faible volume

On s'attend à un rendement plus élevé, avec un courant seuil réduit, en utilisant une simple ou double hétérostructure formée de matériaux semiconducteurs différents. Ces matériaux sont caractérisés par des indices de réfraction et des largeurs de bande interdite $E_{g}$ différents, favorisant le confinement à la fois des porteurs et des photons. Ce confinement renforcé des porteurs entraîne une diminution sensible du courant seuil de la diode.

Le courant seuil est ainsi réduit à $2.000 \mathrm{~A} / \mathrm{cm}^{2}$ pour une $\mathrm{DL}$ à double hétérojonction, contre $8.000 \mathrm{~A} / \mathrm{cm}^{2}$ pour une DL à simple hétérostructure et $40.000 \mathrm{~A} / \mathrm{cm}^{2}$ pour les lasers à homojonction. Techniquement, on peut encore réduire ce courant seuil en réduisant la section conductrice de la diode à une valeur l' (Fig. 7).

La diode laser $\mathrm{GaAs} / \mathrm{GaAl}_{\mathrm{x}} \mathrm{As}_{1-\mathrm{x}}$ est la plus utilisée car, elle peut facilement être dopée $\mathrm{n}$ ou $\mathrm{p}$, et de plus le composé ternaire assure un accord de maille $(0.1 \%)$ presque parfait avec $\mathrm{GaAs}$, quelque soit la composition $\mathrm{x}$. On réduit ainsi les contraintes à l'interface et par voie de conséquence les défauts dans la structure. La Fig. 7 représente la structure d'une diode laser à double hétérojonction:

- la zone active GaAs(p) est une couche de faible épaisseur $(\sim 0.2 \mu \mathrm{m})$ en sandwich entre deux couches de $\mathrm{GaAl}$ AS et GaAs dopées n et $\mathrm{p}$ de $1 \mu \mathrm{m}$ d'épaisseur

- ces hétérojonctions constituent une barrière de potentiel au passage des porteurs, d'où un confinement des porteurs dans la zone active

Le confinement des électrons et trous dans le schéma des bandes et la variation d'indice sont visualisés Fig. 8.

Pour les télécommunications optiques, on préfère utiliser les diodes lasers à la place des DEL, vu leur brillance plus élevée et leur plus faible divergence, ce qui assure un meilleur couplage aux fibres optiques.

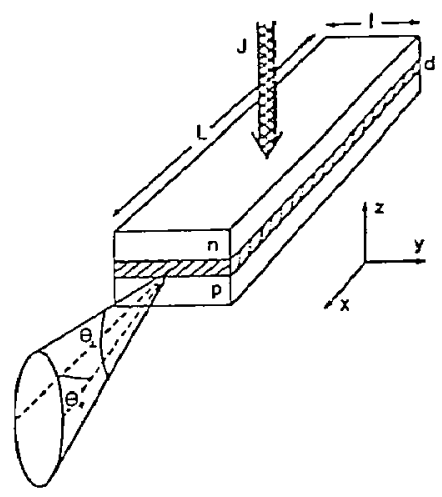

Fig. 6: Diode laser à homojonction

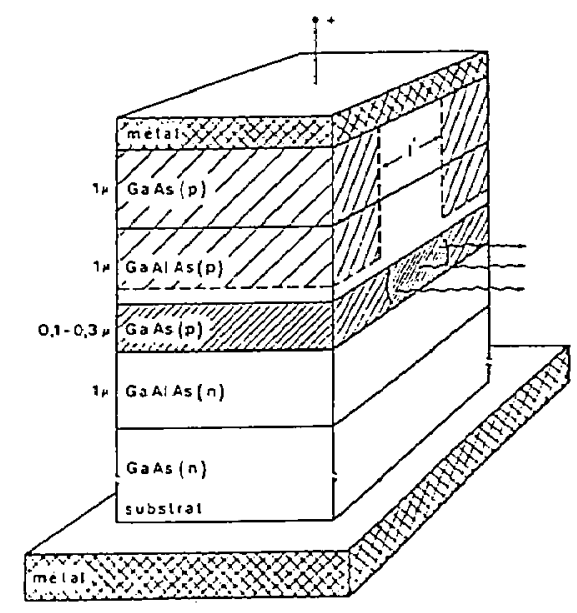

Fig. 7: Diode laser à double hétérojonction 
- la diode laser précédente (basée sur GaAlAs avec $\mathrm{x}=0.7$ ) émet dans l'infrarouge 0.9 $\mu \mathrm{m}$. Or, les télécommunications optiques requièrent une émission dans les fenêtres de transparence des fibres optiques, à savoir 1.1 et $1.6 \mu \mathrm{m}$.

- cette condition est réalisée à l'aide d'une double hétérostructure basée sur le composé quaternaire $\mathrm{Ga}_{\mathrm{x}} \mathrm{In}_{1-\mathrm{x}} \mathrm{P}_{\mathrm{y}} \mathrm{As}_{\mathrm{l-y}}$ qui émet à $1.3 \mu \mathrm{m}$ (pour $\mathrm{x}=0 ; 27$ et $\mathrm{y}=0.6$ ) ou $1,55 \mu \mathrm{m}$ en variant la composition.

A l'heure actuelle, on dispose de diodes lasers commerciales émettant dans une gamme spectrale allant de l'infrarouge au jaune. La variation de la composition $\mathrm{x}$ de ces composés permet en effet d'ajuster la bande interdite du semiconducteur et donc la couleur de la lumière émise.

\subsection{Diodes lasers à puits quantiques}

L'optoélectronique a été révolutionnée vers 1975 par l'apparition de structures nouvelles à caractère quantique, où les électrons et trous sont confinés dans des puits quantiques. Le confinement des électrons et des trous dans ces structures 2D (où un degré de liberté est bloqué dans une direction) entraîne la quantification des niveaux électroniques dans les puits de potentiel. Ces puits quantiques présentent de nombreux avantages par rapport aux structures classiques:

- la raie émise par une transition entre deux niveaux discrets a une largeur spectrale beaucoup plus faible que celle émise par une transition bande à bande dans une diode laser classique

- l'écart d'énergie $\Delta \mathrm{E}$ entre deux niveaux quantiques est ajustable en fonction de la largeur $L$ du puits, ce qui permet d'accorder la longueur d'onde de la lumière émise

- les propriétés physiques des porteurs et des excitons sont fortement modifiées par le confinement quantique (mobilité renforcée, énergie de liaison des excitons augmentée...).

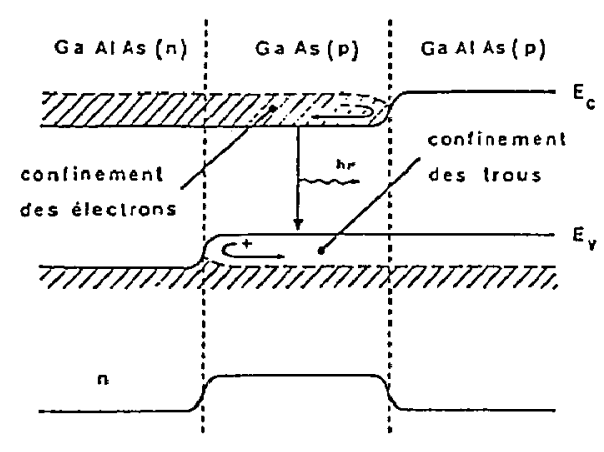

Fig. 8: Confinement des porteurs dans une diode laser de GaAs

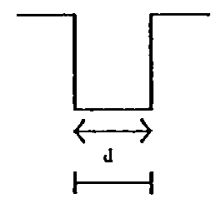

(4)

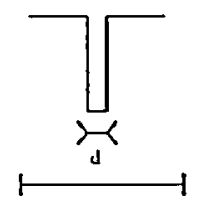

(b)

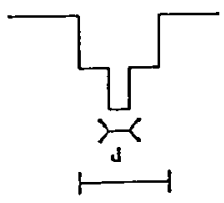

(c) $\longleftrightarrow \quad$ largeur de la couche active $\vdash$ confinement optique

Fig. 9: Schéma d'une hétérostructure a) classsique b) à puits quantiques c) $\mathrm{SCH}$

\subsubsection{Diodes lasers à puits quantique unique}

On a vu que le courant seuil $J_{s}$ des $D L$ diminue si l'épaisseur de la couche active est réduite. Dans les DL classiques cette épaisseur varie de 0.1 à $03 \mu \mathrm{m}$ et ne conduit qu'à un 
confinement partiel des porteurs. Si l'épaisseur $\mathrm{d}$ de la couche active est encore réduite à une valeur inférieure à $20 \mathrm{~nm}$, (telle que $\mathrm{d} \ll \mathrm{L}_{\mathrm{d}}$, longueur de diffusion des électrons), alors le confinement des porteurs dans la zone active est total.

Mais, cette réduction de la zone active a un effet néfaste sur le confinement optique des puits quantiques: le facteur de confinement optique est plus petit que $1(\Gamma<1)$. Or, comme le courant seuil $\mathbf{J}_{8}$ varie en $1 / \Gamma$, celui-ci augmente donc si le confinement optique est diminué. On remédie à cette dégradation du confinement optique dans un puits quantique, soit en modifiant la géométrie de ce puits ou soit en utilisant des puits quantiques multiples: on distingue alors entre les diodes lasers à confinement séparé (diodes SCH ou diodes GRINSCH à gradient d'indice ou les diodes à puits quantiques multiples MQW).

Ces nouvelles structures (Fig.9) permettent donc de séparer spatialement le confinement des porteurs (dans la zone active) et le confinement des photons (zone élargie): on améliore ainsi la valeur du facteur $\Gamma$ et donc le courant seuil. Exemples:

- ainsi pour les lasers GaAs/GaAlAs, les densités de courant seuil sont de $600 \mathrm{~A} / \mathrm{cm}^{2}$ pour les lasers $\mathrm{SCH}$ et de $400 \mathrm{~A} / \mathrm{cm}^{2}$ pour les lasers GRINSCH

\subsection{Diodes lasers dans le bleu et proche UV}

La mise au point de diodes émettant à plus courtes longueurs d'onde (c.à.d le bleu ou le proche UV) est encore en pleine phase de recherche et nécessite l'utilisation d'autres semiconducteurs à plus large bande interdite $\left(\mathrm{E}_{\mathrm{g}}>2.5 \mathrm{eV}\right)$. Mais, la technologie de fabrication de ces couches minces de $\mathrm{ZnSe}$ ou GaN est encore loin d'être maîtrisée pour ces composés car elle se heurte encore à de sérieux problèmes de matériaux, à savoir [7]:

- une difficulté intrinsèque de dopage de ces composés due à une auto-compensation des défauts dans les matériaux à large bande interdite (ex. le dopage $\mathrm{p}$ de $\mathrm{ZnSe}$ ou le dopage $\mathrm{n}$ de $\mathrm{ZnTe} . .$.

- la relative mauvaise qualité cristalline de ces couches se traduisant par un grand nombre de défauts (de $10^{6}$ à $10^{10} \mathrm{~cm}^{-3}$ )

- la mise au point d'alliages ternaires ou quaternaires de ces composés (ex. ZnSSe, $\mathrm{ZnTeSe}, \mathrm{ZnCdSe}, \mathrm{ZnMgSSe}$ ou $\mathrm{AlGaN}, \mathrm{InGaN} . .$. ) capables d'assurer un accord de maille avec le substrat et de réduire les problèmes d'interfaces

- la difficulté de réaliser de bons contacts ohmiques sur ces composés

Ces problèmes de matériaux limitent encore fortement le développement et les performances de ces nouvelles diodes lasers. Néanmoins, des progrès sensibles ont été obtenus à partir des années 1990, à la fois dans la croissance épitaxiale de ces composés et le développement d'alliages adaptés. Ces recherches ont finalement abouti à la mise au point des premiers prototypes lasers basés sur $\mathrm{ZnSe}$ et $\mathrm{GaN}$ [10].

- en 1991, la Société 3M Research Lab. et les Universités Brown et Perdue (USA) ont démontré pour la première foisun effet laser, à basse température et en régime pulsé, avec des diodes $\mathrm{ZnSe} / \mathrm{ZnCdSe}$ et $\mathrm{ZnSSe} / \mathrm{ZnCdSe}$ à double hétérostructure épitaxiées sur un substrat GaAs.

- de 1991 à 1995, de nouveaux progrès ont permis de faire fonctionner ces lasers à 300 $K$ en régime pulsé, puis en régime continu avec des temps de fonctionnement croissant de 1 s à quelques heures.

- En 1996, une nouvelle percée due à la société Nichia Co. (Japon) a conduit à la démonstration d'un effet laser à $300 \mathrm{~K}$ et en mode pulsé, avec une double hétérostucture à base de GaN et AlN. 
La course pour la réalisation d'une diode laser commerciale dans le bleu (ou le proche UV) basée sur les composés $\mathrm{ZnSe}$ ou GaN est donc ouverte. Les Laboratoires européens sont également bien engagés dans cette compétition, ce qui ressort en particulier de leur participation à la dernière Conférence du E-MRS Spring Meeting en 1996 à Strasbourg [11].

\section{CONCLUSION}

Le marché des diodes à semiconducteurs DEL ou DL est en pleine croissance et supporté par des applications existantes ou potentielles de plus en plus nombreuses. D'autre part, les progrès constants en sciences des matériaux ont permis la réalisation de structures nouvelles plus complexes et aussi plus performantes [12]. La commercialisation de diodes performantes dans le bleu et le proche UV peut être raisonnablement envisagée avant l'an 2000, ouvrant ainsi des perspectives nouvelles.

Les applications potentielles de ces diodes concernent ainsi, le stockage optique avec des densités de stockage prévisible de $10 \mathrm{Gbits} / \mathrm{in}^{2}$ (pour $500 \mathrm{Mbits} / \mathrm{in}^{2}$ actuellement en stockage magnétique), mais aussi et surtout l'immense marché de l'affichage optique en couleurs. Une autre application est susceptible de révolutionner à moyen terme le domaine de l'éclairage domestique et public, en produisant de la lumière blanche à partir des trois couleurs de base (rouge-vert-bleu) grâce à des diodes électroluminescentes, et ceci avec une économie en énergie prévisible de $80 \%$ par rapport aux lampes à incandescence.

\section{Références}

[1] J.W. Cook et J.F. Schetzina, Laser Focus World, mars (1995) 101-104

[2] L. Marshall , Laser Focus World, février (1996) 87-94

[3] R.L. Gunshor et A.V. Nurmiko, Laser Focus World, mars (1995) 97-100

[4] H. Haase , J. Qiu , J. De Puydt et H. Cheng, Appl. Pys. Lett., 59 (1991) 1272

[5] H. Mathieu , Physique des semiconducteurs et des composants électroniques,Masson

[6] B. Mombelli, Processus optiques dans les solides,Masson

[7] H. Luo et J.K. Furdyna, Semicond. Sci. Tecnol., 10 (1995) 1041-1048

[8] J. Hecht, Laser Focus World, mars (1996) 53-54

[9] S. Nakamura et al. , Jpn. J. Appl. Phys., 34 (1995) L1332

[10] S. Nakamura, Jpn. J. Appl. Phys., 35 (1995) L74

[11] UV,Blue and Green Light Emission from Semiconductor Materials, E-MRS Spring Meeting in Strasbourg, France (1996) to be published in Appl. Surface Science

[12] C. Weisbuch, J. of Crystal Growth, 138 (1994) 776-785 